This is an electronic reprint of the original article. This reprint may differ from the original in pagination and typographic detail.

Author(s): Rautiala, Petri; Helanterä, Heikki; Puurtinen, Mikael

Title: $\quad$ The evolutionary dynamics of adaptive virginity, sex-allocation and altruistic helping in haplodiploid animals

Year: $\quad 2018$

Version:

Please cite the original version:

Rautiala, P., Helanterä, H., \& Puurtinen, M. (2018). The evolutionary dynamics of adaptive virginity, sex-allocation and altruistic helping in haplodiploid animals. Evolution, 72(1), 30-38. https://doi.org/10.1111/evo.13399

All material supplied via JYX is protected by copyright and other intellectual property rights, and duplication or sale of all or part of any of the repository collections is not permitted, except that material may be duplicated by you for your research use or educational purposes in electronic or print form. You must obtain permission for any other use. Electronic or print copies may not be offered, whether for sale or otherwise to anyone who is not an authorised user. 


\title{
THE EVOLUTIONARY DYNAMICS OF ADAPTIVE VIRGINITY, SEX- ALLOCATION AND ALTRUISTIC HELPING IN HAPLODIPLOID ANIMALS
}

\author{
Petri Rautiala ${ }^{1, *}$ (petri.rautiala@gmail.com) \\ Heikki Helanterä ${ }^{2,3}$ (heikki.helantera@helsinki.fi) \\ Mikael Puurtinen ${ }^{3,4}$ (mikael.puurtinen@jyu.fi)
}

1 School of Biology, University of St Andrews, St Andrews KY16 9TH, United Kingdom

2 University of Helsinki, Department of Biosciences, PO Box 65, FI-00014 University of Helsinki, Finland

3 Centre of Excellence in Biological Interactions

$4 \quad$ University of Jyvaskyla, Department of Biological and Environmental Science, PO Box 35, FI40014 University of Jyvaskyla, Finland

* Corresponding author

Keywords: Alternative reproduction strategies, mating behavior, reproductive altruism, split sex ratios, virgin reproduction.

\begin{abstract}
In haplodiploids, females can produce sons from unfertilized eggs without mating. However, virgin reproduction is usually considered to be a result of a failure to mate, rather than an adaptation. Here we build an analytical model for evolution of virgin reproduction, sex-allocation, and altruistic female helping in haplodiploid taxa. We show that when mating is costly (e.g. when mating increases predation risk), virginity can evolve as an adaptive female reproductive strategy. Furthermore, adaptive virginity results in strongly divergent sex-ratios in mated and virgin queen nests ('split sex
\end{abstract}

This article has been accepted for publication and undergone full peer review but has not been through the copyediting, typesetting, pagination and proofreading process, which may lead to differences between this version and the Version of Record. Please cite this article as doi: 10.1111/evo.13399.

This article is protected by copyright. All rights reserved. 
ratios'), which promotes the evolution of altruistic helping by daughters in mated queen nests. However, when helpers evolve to be efficient and increase nest production significantly, virgin reproduction is selected against. Our results suggest that adaptive virginity could have been an important stepping stone on the pathway to eusociality in haplodiploids. We further show that virginity can be an adaptive reproductive strategy also in primitively social haplodiploids if workers bias the sex ratio toward females. By remaining virgin, queens are free to produce sons, the more valuable sex in a female-biased population. Our work brings a new dimension to the studies linking reproductive strategies with social evolution.

\section{Introduction}

In most sexual systems, individuals cannot reproduce without mating at least once. However, in haplodiploids, where females are born from fertilized eggs and males from unfertilized eggs, females can reproduce also without mating, albeit then being constrained to produce only sons. This type of virgin reproduction has been seen as an option of last resort when a female fails to mate or obtain viable sperm (Godfray 1990; Schmidt et al. 2014). With the exception of Tilquin (2015), there is no formal theory of virgin reproduction as an adaptive reproductive strategy of haplodiploid females except in the context of selfish worker reproduction (Ratnieks et al. 2006; Wenseleers and Ratnieks 2006; Alpedrinha et al. 2013).

The high prevalence of non-reproductive female helpers in haplodiploid Hymenoptera spurred a series of influential studies on the role of relatedness asymmetries in evolution of altruistic helpers (e.g. Hamilton 1964; Hamilton 1972). Later refinements to the theory found that haplodiploidy promotes female helping behavior only when there is variation in sex ratio among nests ('split sex ratios'; Trivers and Hare 1976; Grafen 1986). When helpers are in nests where the sex ratio is more female biased than the population sex ratio, female helpers value an average sibling more than an average offspring. At the origin of helping, the only plausible factor giving rise to split sex ratios is virgin reproduction, where unmated females produce progeny of all males, and mated females are selected to produce female biased broods, promoting evolution of female helping (Gardner et al. 2012; Alpedrinha et al. 2013).

However, because virgin reproduction is considered rare in extant taxa, it has been argued that haplodiploidy has had no appreciable role in the evolution of altruistic helping (Gardner et al. 2012). Yet, previous models have not considered virgin reproduction as an adaptive reproductive strategy (Godfray 1990; Gardner et al. 2012; Rautiala et al. 2014). If selection would favor high frequency of virgin reproduction, this could lead to strongly split sex ratios, promoting the evolution of female helping. Indeed, it has been suggested that risks associated with mating (e.g. exposure to predators or pathogens; Whitcomb et al. 1973; Daly 1978; Wing 1988; Helms et al. 2016) could select for virginity as an alternative reproductive strategy for haplodiploid females (Godfray and Hardy 1993; Godfray and Cook 1997; Tilquin 2015).

This article is protected by copyright. All rights reserved. 
Here we develop an analytical model to study the evolution of virginity in an environment where mating carries a risk, and find the requirements for virginity to evolve as an adaptive reproductive strategy. We link adaptive virginity to female helping behavior by analyzing how the benefit requirements for altruistic helping change via elevated in-nest relatedness when virginity strategy evolves. Our analysis shows that adaptive virginity offers a unique adaptive pathway to sociality in haplodiploids not plausible for diplodiploid taxa. We further show that virgin reproduction can be selected also after helping has evolved, if helpers take control of sex-ratio and shift the population sex ratio towards females (Trivers and Hare 1976; Helanterä and Ratnieks 2009). If population sexratio is female biased, queens will value sons more than daughters. In such circumstances, reproducing as a virgin and producing only sons can be more beneficial than mating and producing more offspring (mostly daughters) with helpers. To guide future experimental research, we discuss life-history and mating system features that are likely to affect virgin reproduction.

\section{Model and results}

From here on, we refer to any successfully reproducing female as a 'queen'. An altruistic daughter who stays in her natal nest to rear her siblings without pursuing own reproduction is referred to as a 'helper' or 'female helper'. The required benefits for helping to be favored are calculated for daughters only, and the promoting factors derived in our analysis only promote female helping behavior. Taxa where helping has not evolved are termed 'non-social', and taxa where all mated queens have female helpers are termed 'social'. In social taxa, the helpers are born first, and they are not taken into account in any mention or parameter of nest production, sex allocation, sex ratio, or class of relatives. Sons and daughters cost the same to produce, and sex ratio adjustment is costless for both the queen and the helpers.

In our model, we analyze how the state of the population (presence/absence of helpers, virginity tendency, mated queen sex ratio, and requirements for the evolution of altruistic helping) changes when selection is at work. The derivations are done entirely in the Supporting Information, and only the results are presented and explained here. Instead of specifying the genetic mechanisms underlying phenotypes, we use the 'phenotypic gambit' and assume that selection acts directly on phenotypes (Grafen 1984). For a more specific genetic model on virgin reproduction see Tilquin (2015). Even though we analyze the evolution of virgin reproduction and mated female sex ratios together, we discuss their evolution as if the traits evolved sequentially in order to clarify the selective forces which are binding the traits together.

\section{Model assumptions}

We analytically compare the inclusive fitness consequences of different behavioral strategies, while taking into account how those consequences are affected by the choices made by the rest of the population (Hamilton 1964; Taylor 1996; Taylor and Frank 1996). The stable reproductive and sex allocation strategies are found by analyzing whether selection favors a mutant whose strategy differs from the population average (Taylor and Frank 1996). The number of new siblings that needs to be reared for helping to be beneficial for a potential female helper $\left(b_{\mathrm{tr}}\right.$, termed 'benefit

This article is protected by copyright. All rights reserved. 
threshold') is solved by comparing the expected inclusive fitness outcome of pursuing own reproduction to that of remaining as a helper (Taylor 1996; for details see SI Part A). Model variables and symbols are given in Table 1.

We consider a finite, panmictic population with discrete, non-overlapping generations. Mating females produce offspring with only one male. The population is sufficiently large so that the actions of a single individual have a negligibly small effect on population level parameters. With probability $u$, females reproduce as virgin and are constrained to produce only sons. Mating incurs a mortality risk, with fraction $s$ of mated females surviving. In the Discussion, we explain how model results apply also to mating costs that reduce female fecundity, instead of mortality risk. The mortality risk is considered constant and is not affected by changes in other population parameters.

The number of breeding positions in each generation is limited to $n$, for which surviving females born in the previous generation compete. Virgin queens of both non-social and social taxa produce $N$ offspring (only sons). Mated queens produce $k \times N$ reproductive offspring. The 'production amplifier' $k$ captures the possible effects of mating on number of offspring, and in social taxa, also the impact of helpers on nest productivity. In social taxa, the production amplifier $k$ is a combination of both the helper numbers and the efficiency of individual helpers. We are not separating the two in this analysis, and growth in the production amplifier can be a result of a rise in either of these parameters. The 'production amplifier' $k$ is assumed to be constant when we analyze evolution of virginity tendency $(u)$ and mated queen sex ratio (proportion of sons, $z$ ). The population sex ratio (proportion of males among all reproductive offspring) is denoted by $\hat{z}$.

\section{Optimal sex ratio strategy from the mated queen's perspective}

The optimal sex ratio strategy for the mated queens depends on the proportion of virgin queens in the population (Godfray and Grafen 1988; Godfray 1990). Virgin queens produce only sons, and mated queens are selected to react to this by biasing their offspring sex ratio towards daughters. In haplodiploids, queens can easily adjust their produced sex ratio by controlling the proportion of eggs they fertilize. The evolutionary attractor for the mated queen sex ratio is derived in SI Part B and equals

$$
z_{\mathrm{q}}(u)=\left\{\begin{array}{rl}
\frac{(1-u) s \times k-u}{2(1-u) s \times k}, & u<\frac{s \times k}{s \times k+1} \\
0, & u \geq \frac{s \times k}{s \times k+1}
\end{array},\right.
$$

which results in a population sex ratio (census of juveniles in each generation before any matingrelated mortality)

$$
\hat{z}_{\mathrm{q}}(u)=\left\{\begin{aligned}
\frac{1}{2}, & u<\frac{s \times k}{s \times k+1} \\
\frac{u}{(1-u) s \times k+u}, & u \geq \frac{s \times k}{s \times k+1}
\end{aligned}\right.
$$

This article is protected by copyright. All rights reserved. 
As long as the mated queens are able to shift their produced sex ratio toward daughters at increasing frequencies of virgins, the population sex ratio remains at one half. Once the frequency of virgins rises to a level where mated queens are selected to produce only daughters, the population sex ratio becomes male biased. Selection on queen-controlled sex ratio, together with selection on virginity tendency, is depicted in Figure 1 panels $C$ and $D$.

\section{Evolution of adaptive virginity and queen-controlled sex ratios}

When the queen is in control of the sex-ratio of her brood, selection favors virginity when risks of mating are so high that the expected number of offspring is lower for mated than for virgin queens $(s \times k<1$; SI Part C). Under queen-controlled sex-ratio, the evolutionary attractor for virginity tendency is

$$
u_{\mathrm{q}}=\left\{\begin{array}{ll}
\frac{1}{2}, & s \times k<1 \\
0, & s \times k>1
\end{array}, \quad\right. \text { 3] }
$$

and is illustrated in Figure 1A. When the frequency of virgin queens increases, mated queens are selected to compensate the surplus of males by biasing their offspring production to females (Fisher 1930; Godfray 1990) as presented above in equation 1. From equation 1, we see that at the non-zero virginity tendency $u_{\mathrm{q}}=1 / 2$, mated queens produce all-daughter broods. In this stable state, the population reproductive offspring sex ratio is male biased $\left[\hat{z}_{q}(1 / 2)=1 /(s \times k+1)\right]$, in contrast to the verbal argumentation by Godfray and Hardy (1993) who suggested that virginity can only be selected for as long as the population sex ratio remains at 1:1. The reason behind this is explained in the Discussion.

\section{Virginity promotes helping via split sex ratios}

As virginity tendency increases and mated queens respond by producing more females, female offspring experience an elevated relatedness within their natal nest. This elevated average in-nest relatedness is not countered by lowered reproductive value of sisters, as the population sex ratio is less female biased than the sex ratio in mated-queen nests ('split sex ratios'-effect; Grafen 1986). In the absence of virgin queens, regardless of the magnitude of the offspring production $N$, a helper needs to rear more than 2 new siblings for it to be more beneficial than pursuing own reproduction ('benefit threshold': SI Part D). With increasing frequency of virgins, the benefit threshold $\left(b_{\mathrm{tr}}\right)$ for helping decreases until it reaches the value of $4 / 3$

$$
b_{\mathrm{tr}}(u)=\left\{\begin{aligned}
\frac{4(1-u) s \times k}{2(1-u) s \times k+u}, & u<\frac{s \times k}{s \times k+1} \\
\frac{4}{3}, & u \geq \frac{s \times k}{s \times k+1} .
\end{aligned}\right.
$$

At the evolutionary attractor value for virginity tendency $u=1 / 2$ (SI Part C), the benefit threshold for helping is $4 / 3$ due to split sex ratios and the elevated relatedness in the nests of mated queens (equation 4), making evolution of helping easier. The lowest threshold of $4 / 3$ is reached already when $u=s \times k /(s \times k+1)$, the same point where mated queens start to produce all daughter broods

This article is protected by copyright. All rights reserved. 
(dashed line in Figure 1A; plateau starting points in Figure 2). If some unknown factor is preventing virginity tendency from achieving its expected level of $1 / 2$, the promoting effect can still be in its full potential (Figure 2).

\section{High impact of helpers on nest production selects against virginity}

If helping evolves to a point where the expected number of offspring for mating queens exceeds that for virgins ( $s \times k>1$, equation 3 ), virginity is selected against. With the loss of virgin queens, split sex ratios are also lost, and altruistic helping is no longer promoted. Without virgin queens, the queencontrolled sex ratio returns to one half, and the benefit threshold returns to 2 (equation 4). Unless the efficiency of individual helpers evolved to a high enough level $(b>2)$ during the period when helpers were present, the disappearance of virgin reproduction can decrease or even eradicate helping behavior from the population. This would lead the population into a state where virginity and helping behavior are not stably established. Only if the efficiency of individual helpers evolves so that the benefit threshold of 2 is satisfied and the total impact of helpers satisfies $s \times k>1$, does the population escape to a state where virginity is no longer selected for and helping behavior is stably established.

\section{Virginity tendency can be selected for when helpers control the sex ratio}

So far we have analyzed the evolution of virgin reproduction and its effects on the evolution of helping when queens are in full control of sex ratios. Next, we analyze selection on virgin reproduction when helpers have taken control of the nest sex ratio. This analysis does not assume that helping necessarily evolved via the route of adaptive virginity. We assume that helper control of sex ratio evolves when virginity is absent. This is because when virginity is common, helpers are in nests that only produce females, and there is no opportunity to bias the sex-ratio.

Female helpers in haplodiploid taxa prefer a more female biased sex ratio than the queen (Trivers and Hare 1976). This is because female helpers are more related to their full sisters than to their brothers. If the helpers control their sibling sex ratio (Sundstrom et al. 1996; Hammond et al. 2002; Helanterä and Ratnieks 2009), the evolutionary attractor for sex ratio in the nests of mated females is (derived in SI Part B)

$$
Z_{\mathrm{h}}(u)=\left\{\begin{aligned}
\frac{(1-u) s \times k-3 u}{4(1-u) s \times k}, & u<\frac{s \times k}{s \times k+3} \\
0, & u \geq \frac{s \times k}{s \times k+3}
\end{aligned}\right.
$$

which results in a population sex ratio of

$$
\hat{z}_{\mathrm{h}}(u)=\left\{\begin{aligned}
\frac{1}{4}, & u<\frac{s \times k}{s \times k+3} \\
\frac{u}{(1-u) s \times k+u}, & u \geq \frac{s \times k}{s \times k+3} .
\end{aligned}\right.
$$

This article is protected by copyright. All rights reserved. 
If the helpers win the sex ratio conflict and shift the sex ratio to their optimum as presented by equations 5 and 6 , the female biased population sex ratio elevates the value of sons and decreases the value of daughters for the queen. By remaining a virgin, a female then not only avoids the risks of mating, but also the sex ratio manipulation by her helpers: she is free to produce offspring of the more valuable sex (sons), even if at a smaller quantity. In SI Part C, we find that under helpercontrolled sex ratio, the evolutionary attractor for virginity tendency is

$$
u_{\mathrm{h}}=\left\{\begin{array}{rr}
1 / 2, & s \times k<2 \\
0, & s \times k>3
\end{array}\right.
$$

(illustrated in Figure 1B). At the non-zero strategy of $u_{\mathrm{h}}=1 / 2$, mated queens produce all-daughter broods, and in the absence of virgin queens the mated queens produce $1 / 4$ sons (Trivers and Hare 1976).

Within range $2 \leq s \times k \leq 3$ there are two evolutionary attractors for virginity tendency. Within this range, selection increases virginity tendency when $(s \times k-2) /(s \times k-1)<u_{\mathrm{h}}<1 / 2$, and decreases it otherwise (SI Part C). In the $2 \leq s \times k \leq 3$ range, $u_{h}=1 / 2$ and $u_{h}=0$ are evolutionary attractors for virginity tendency and $u_{\mathrm{h}}=(s \times k-2) /(s \times k-1)$ is an evolutionary repeller between the two attractors (Geritz et al. 1998). Above the evolutionary repeller, the helpers allow their queen to produce so few valuable sons that, even when combined with the high number of daughters, remaining a virgin is more beneficial. Below the repeller, the queen is allowed to produce enough sons to elevate the inclusive fitness returns of mating above that of remaining a virgin. This result means that virgin reproduction can remain stably established at higher values of helper impact on production $(k)$ than where it could evolve de novo. More precisely, if virginity tendency evolves to $u_{\mathrm{h}}=1 / 2$ when $s \times k<2$, it drops to zero only when the productivity of the mated queen nests increases so that $s \times k>3$. The repeller is illustrated as a hollow line in Figure $1 \mathrm{~B}$, and the evolutionary trajectories of virginity tendency and helper-controlled sex ratio under fixed parameters $s$ and $k$ are illustrated in Figure $1 \mathrm{~F}$.

Virginity has no effect on the benefit threshold under helper controlled sex ratios (SI part D, equation S10).

\section{Discussion}

\section{Adaptive virginity in non-social taxa}

We have shown that remaining virgin can be an adaptive reproductive strategy for a haplodiploid female when risks of mating (e.g. predation or pathogen pressure) reduce the number of offspring a female expects to have. By remaining virgin, a female avoids mating-associated risks, but is constrained to produce only sons. At equilibrium, $50 \%$ of queens should reproduce as virgin, and mated queens should produce only daughters. Since, due to the risks of mating, the expected number of offspring is less for mated than for virgin queens, at equilibrium the population sex ratio is male-biased.

Why doesn't selection for virginity stop when the population sex ratio is even, and why is the nonzero equilibrium for virginity one half, regardless of the magnitude of risks of mating? The answer 
can be found in Fisherian sex allocation theory (Fisher 1930). When mated females produce only daughters, virginity is synonymous with sex allocation strategy. Probability of remaining virgin equals females' probability of producing only sons, and probability of mating equals probability of producing only daughters. In other words, sex allocation is equal to probability of mating. As sex allocation theory predicts, the equilibrium is found at one half (Fisher 1930). This interpretation is supported by the notion that virginity is selected to drop from one half to zero exactly at the point where the mated queens start to produce also sons. This breaking point is illustrated in Figures $1 \mathrm{~A}$ and $1 \mathrm{~B}$ as a dashed line (from the domain limits of equations 1 and 5 respectively), above which mated queens are producing only daughters, i.e. where virginity and sex allocation are synonymous. If the mated queens' sex allocation strategy would be biased, for example due to local mate or resource competition, so would the strategy for virginity tendency (unpublished results). We leave the analysis of these effects for future studies, as it would be too cumbersome to reproduce here, and would not add value to the presented results. Our results show that the verbal argument by Godfray and Hardy (1993), that virginity can be selected until the population sex ratio reaches 1:1, does not hold. Rather, virginity is selected until the reproductive-strategy determined sex allocation reaches 1:1.

A simulation by Tilquin (2015) resulted in half of the females becoming homozygous for a recessive allele which causes the female to remain a virgin, i.e. the same conclusion as drawn from our analytical model that the evolutionary attractor for virginity tendency strategy is one half. However, in Tilquin (2015) mating entailed an energy cost which lowered the fecundity of mated queens, whereas in our model the cost of mating is elevated mortality. Our model could be modified to reflect the assumptions of Tilquin (2015) by setting the mortality cost of mating to zero $(s=1)$ and by setting the value of the production amplifier parameter $k$ to be less than one, so that mated females produce fewer offspring than virgin females. This same modification could be used to include any mechanism that might lower the fecundity of mated females.

\section{Adaptive virginity and the evolution of helping}

By analyzing the feedbacks between adaptive virginity, facultative helping behavior, and mated queen sex ratio, we have shown how haplodiploidy offers a unique adaptive pathway to evolution of helping. The presence of virgins promotes the emergence of female altruism in haplodiploids via 'split sex ratios', as already noted by Godfray and Grafen (1988), and further analyzed by Gardner et al. (2012) and Rautiala et al. (2014). Our analysis expands this literature by showing that virgin reproduction can be adaptive, and that selection favors high levels of virginity. Therefore, transitional stages of high levels of adaptive virginity could have been important at the initial stages of sociality in haplodiploid taxa. Interpretations based on low levels of virginity in present taxa may thus have underestimated the role of split sex ratios in promoting the evolution of helping in haplodiploids (Gardner et al. 2012). However, it should be noted that virgin reproduction can also hinder the transition to more advanced forms of sociality. Since virgin queens do not have helpers, selection in virgin nests does not favor queen specialization on egg production. The queens face

This article is protected by copyright. All rights reserved. 
antagonistic selection pressures: genetically determined increases in egg-laying and decreases in provisioning of offspring can be selected for in mated queens, but selected against in virgin queens.

If female helpers take control of mated queen sex-ratio and shift it toward females, virgin reproduction can be adaptive also in social taxa. When the population sex ratio is female biased, sons are more valuable to the queen than daughters. By remaining virgin, a queen is free from helper manipulation and can produce an all-son brood, even if at a smaller quantity. Virginity can evolve de novo when the expected reproductive output of mated queens is less than twice that of virgin queens $(s \times k<2)$, and is locally stable when mated queen output is less than three times higher than virgin output $(s \times k<3)$. Selection for virginity in social taxa does not require that helping originated via adaptive virginity, but occurs whenever helpers control of the sex-ratio and the ratio of expected productivity of mated and virgin queens is sufficiently low.

Evolution of adaptive virginity in social taxa requires that helpers evolve to bias the sex ratio before the productivity benefit of having helpers reaches high levels $(s \times k<2)$. Female biased sex ratios and adaptive sex ratio manipulation by helpers in primitively eusocial species (Mueller 1991; Boomsma and Eickwort 1993; Packer and Owen 1994) suggests this could be the case. Any cost of sibling sex ratio biasing by helpers, through e.g. efficiency costs of reallocation or recognition errors, is paid from the total offspring production by mated queens (Chapuisat et al. 1997), bringing it closer to the offspring production of virgin queens by lowering $k$. This feedback makes it even more plausible that the condition $(s \times k<2)$ is fulfilled. The search for both sex ratio biasing and adaptive virginity should be focused on primitively eusocial taxa not only for low $k$ values, but also for queens possibly having retained their ability for solitary reproduction as virgins.

\section{Is there adaptive virginity in nature?}

The model predicts very high frequencies (50\%) of adaptive virginity in haplodiploids under suitable conditions. Such extreme levels of virginity have not been reported from nature, but there is evidence of substantial frequencies of females voluntarily remaining virgin. Guertin et al. (1996) found that $10 \%$ of parasitoid wasp Bracon hebetor females refused to mate in their laboratory experiment. In a field experiment by Adams and Morse (2014) on parasitoid wasp Alabagrus texanus, 31 out of $89(34.8 \%)$ females refused to mate with any males they encountered, and remained virgins throughout the experiment. Even though parasitoid wasps do not generally represent good candidates for exploring the connection between social evolution and adaptive virginity, they suggest that virginity can be adaptive. A number of studies also suggest that in species with high prevalence of virgin females, mated females produce highly female-biased sex ratios (Higgins and Myers 1992; Ode et al. 1997; Kranz et al. 2000), in line with the theory (Godfray 1990; Gardner et al. 2012; Rautiala et al. 2014; this paper). This is a prerequisite for virginity to be able to evolve to higher levels, as otherwise the male biased sex ratio caused by virgin reproduction would decrease the relative benefit of remaining virgin. It is important to note that also less extreme frequencies of virginity result in lowered benefit threshold for helping (equation 4; Figure 2), making evolution of helping easier.

This article is protected by copyright. All rights reserved. 
There are a number of reasons why virginity may not reach the levels predicted by the model. Males are selected to counter females' pursuits of remaining virgins in the same way they are selected to obtain more than their fair share of next generation offspring. Adaptive virginity thus brings a new dimension to the sexual conflict over mating between males and females. In some taxa, males compete for control of territories the females need for reproduction (Baker 1983). Another reported male behavior is intercepting females as they are emerging (Alcock et al. 1976, 1977). In the presence of either of these mechanisms it can be difficult for a female to remain virgin by accident. Therefore, in taxa where males control breeding territories or actively intercept females, virginity is predicted to persist only through active adaptations of females to avoid mating. On the other hand, it is more probable that higher levels of virginity are found in taxa where males do not exert dominance over female mating decisions, but in such taxa virginity can also reflect true mating failures.

It should also be noted that virginity will not be selected for in species with frequent sib-mating where females need to produce mixed brood to ensure the reproductive success of the offspring. Sib-mating is common among many haplodiploid taxa (see Tilquin 2015 and references therein), including most taxa where virginity prevalence have been estimated (Godfray and Hardy 1993). As our model assumes panmixis, more data from randomly mating haplodiploid taxa will be necessary to assess model validity.

Finally, it is possible that many observations of virgin reproduction simply go unreported in scientific literature as they are perceived as artifacts of experimental conditions (see Tilquin 2015). Thus, in order to assess whether or not the predictions of our model, and those of Tilquin (2015) are met with empirical data, the possibility of adaptive virginity needs attention as an interpretation for virgin reproduction. Or ideally, experiments specifically designed to detect it should be carried out.

We have shown that adaptive virginity can be an important stepping stone on the pathway to evolution of sociality in haplodiploids. However, we do not claim that adaptive virginity would be the sole explanation for the multiple origins of eusociality in haplodiploids. Instead, we suggest that adaptive virginity could have worked as an additional force, together with specific circumstances such as nest defence and pre-existing maternal care (Queller and Strassmann 1998; Ross et al. 2013), in promoting the evolution of female reproductive altruism.

Our analysis reveals that virginity can reach extreme levels in non-social and primitively social systems, but is selected against when the population reaches advanced forms of sociality. The importance of virginity in the evolution of sociality should thus not be dismissed on the grounds that observed levels of virginity are low in extant taxa. Our work brings a new dimension to the studies linking mating behavior with social evolution that have thus far mainly considered the importance of high relatedness brought by monogamy (Boomsma 2007; Hughes et al. 2008; Boomsma 2009; Cornwallis et al. 2010). Our results suggest new directions for both studying the sexual conflict over mating frequencies, and the evolution of helping behavior in haplodiploids. We urge future experimental studies on haplodiploids to take into account the possibility that observed virginity can be adaptive.

This article is protected by copyright. All rights reserved. 


\section{Acknowledgments}

We thank Eva Kisdi, Andrew Bourke and Stuart West for helpful comments. PR, MP and HH were supported by Academy of Finland (grant 258385 to MP, grant 135970 to $\mathrm{HH}$ and 252411 to the Centre of Excellence in Biological Interactions), PR also by the Royal Society Newton International Fellowship, and $\mathrm{HH}$ also by the Kone Foundation.

\section{References}

Adams, S. A. and D. H. Morse. 2014. Condition-dependent mate choice of a parasitoid wasp in the field. Animal Behaviour 88:225-232.

Alcock, J., C. E. Jones, and S. L. Buchmann. 1976. Location before emergence of the female bee, Centris pallida, by its male (Hymenoptera: Anthophoridae). Journal of Zoology 179:189-199.

Alcock, J., C. E. Jones, and S. L. Buchmann. 1977. Male mating strategies in the bee Centris pallida Fox (Anthophoridae: Hymenoptera). The American Naturalist 111:145-155.

Alpedrinha, J., S. A. West, and A. Gardner. 2013. Haplodiploidy and the evolution of eusociality: worker reproduction. Am Nat 182:421-438.

Baker, R. R. 1983. Insect Territoriality. Annual Review of Entomology 28:65-89.

Boomsma, J. and G. Eickwort. 1993. Colony structure, provisioning and sex allocation in the sweat bee Halictus ligatus (Hymenoptera: Halictidae). Biological Journal of the Linnean Society 48:355-377.

Boomsma, J. J. 2007. Kin selection versus sexual selection: why the ends do not meet. Current Biology 17:R673-683.

Boomsma, J. J. 2009. Lifetime monogamy and the evolution of eusociality. Philosophical Transactions of the Royal Society B-Biological Sciences 364:3191-3207.

Chapuisat, M., L. Sundström, and L. Keller. 1997. Sex-ratio regulation: the economics of fratricide in ants. Proceedings of the Royal Society of London B: Biological Sciences 264:1255-1260.

Cornwallis, C. K., S. A. West, K. E. Davis, and A. S. Griffin. 2010. Promiscuity and the evolutionary transition to complex societies. Nature 466:969-972.

Daly, M. 1978. The cost of mating. The American Naturalist 112:771-774.

Fisher, R. A. 1930. The Genetical Theory of Natural Selection. Clarendon Press, Oxford.

This article is protected by copyright. All rights reserved. 
Gardner, A., J. Alpedrinha, and S. A. West. 2012. Haplodiploidy and the evolution of eusociality: split sex ratios. Am Nat 179:240-256.

Geritz, S. A. H., É. Kisdi, G. Meszéna, and J. A. J. Metz. 1998. Evolutionarily singular strategies and the adaptive growth and branching of the evolutionary tree. Evolutionary Ecology 12:35-57.

Godfray, H. C. J. 1990. The causes and consequences of constrained sex allocation in haplodiploid animals. Journal of Evolutionary Biology 3:3-17.

Godfray, H. C. J. and J. M. Cook. 1997. Mating systems of parasitoid wasps. Pp. 211-225 in J. C. Choe, and B. J. Crespi, eds. The Evolution of Mating Systems in Insects and Arachnids:. Cambridge University Press, Cambridge.

Godfray, H. C. J. and A. Grafen. 1988. Unmatedness and the evolution of eusociality. American Naturalist 131:303-305.

Godfray, H. C. J. and I. C. W. Hardy. 1993. Sex ratio and virginity in haplodiploid insects in D. L. Wrensch, and M. A. Ebbert, eds. Evolution and Diversity of Sex Ratio in Insects and Mites. Routledge, Chapman \& Hall, London.

Grafen, A. 1984. Natural selection, kin selection and group selection. Pp. 62-84 in D. Krebs, and N. B. Davies, eds. Behavioral ecology. BLackwell Scientific Publications, Oxford.

Grafen, A. 1986. Split sex ratios and the evolutionary origins of eusociality. Journal of theoretical biology 122:95-121.

Guertin, D. S., P. J. Ode, M. R. Strand, and M. F. Antolin. 1996. Host-searching and mating in an outbreeding parasitoid wasp. Ecological Entomology 21:27-33.

Hamilton, W. D. 1964. The genetical evolution of social behaviour I \& II. Journal of theoretical biology 7:1-52.

Hamilton, W. D. 1972. Altruism and Related Phenomena, Mainly in Social Insects. Annual Review of Ecology and Systematics 3:193-232.

Hammond, R. L., M. W. Bruford, and A. F. Bourke. 2002. Ant workers selfishly bias sex ratios by manipulating female development. Proceedings of the Royal Society B: Biological Sciences 269:173-178.

Helanterä, H. and F. L. W. Ratnieks. 2009. Sex allocation conflict in insect societies: who wins? Biology Letters 5:700-704.

Helms, J. A., A. P. Godfrey, T. Ames, and E. S. Bridge. 2016. Are invasive fire ants kept in check by native aerial insectivores? Biology Letters 12.

This article is protected by copyright. All rights reserved. 
Higgins, C. J. and J. H. Myers. 1992. Sex ratio patterns and population dynamics of western flower thrips (Thysanoptera: Thripidae). Environmental Entomology 21:322-330.

Hughes, W. O. H., B. P. Oldroyd, M. Beekman, and F. L. W. Ratnieks. 2008. Ancestral monogamy shows kin selection is key to the evolution of eusociality. Science 320:1213-1216.

Kranz, B. D., M. P. Schwarz, L. C. Giles, and B. J. Crespi. 2000. Split sex ratios and virginity in a gallinducing thrips. Journal of Evolutionary Biology 13:700-706.

Mueller, U. G. 1991. Haplodiploidy and the evolution of facultative sex ratios in a primitively eusocial bee. Science 254:442.

Ode, P. J., M. F. Antolin, and M. R. Strand. 1997. Constrained oviposition and female-biased sex allocation in a parasitic wasp. Oecologia 109:547-555.

Packer, L. and R. E. Owen. 1994. Relatedness and sex ratio in a primitively eusocial halictine bee. Behavioral Ecology and Sociobiology 34:1-10.

Queller, D. C. and J. E. Strassmann. 1998. Kin Selection and Social Insects. BioScience 48:165-175.

Ratnieks, F. L. W., K. R. Foster, and T. Wenseleers. 2006. Conflict resolution in insect societies. Annual Review of Entomology 51:581-608.

Rautiala, P., H. Helanterä, and M. Puurtinen. 2014. Unmatedness promotes the evolution of helping more in diplodiploids than in haplodiploids. The American Naturalist 184:318-325.

Ross, L., A. Gardner, N. Hardy, and Stuart A. West. 2013. Ecology, Not the Genetics of Sex Determination, Determines Who Helps in Eusocial Populations. Current Biology.

Schmidt, C. V., S. Frohschammer, A. Schrempf, and J. Heinze. 2014. Virgin ant queens mate with their own sons to avoid failure at colony foundation. Naturwissenschaften 101:69-72.

Sundstrom, L., M. Chapuisat, and L. Keller. 1996. Conditional manipulation of sex ratios by ant workers: A test of kin selection theory. Science 274:993-995.

Taylor, P. D. 1996. Inclusive fitness arguments in genetic models of behaviour. Journal of Mathematical Biology 34:654-674.

Taylor, P. D. and S. A. Frank. 1996. How to make a kin selection model. Journal of theoretical biology 180:27-37.

Tilquin, A. 2015. Why mating? Modelling the spread of a sex-strike allele in haplodiploids. Department of Biology. Master's thesis, Université Claude Bernard Lyon 1.

Trivers, R. L. and H. Hare. 1976. Haplodiploidy and the evolution of the social insects. Science 191:249-263.

This article is protected by copyright. All rights reserved. 
Wenseleers, T. and F. L. W. Ratnieks. 2006. Comparative analysis of worker reproduction and policing in eusocial hymenoptera supports relatedness theory. American Naturalist 168:E163-E179.

Whitcomb, W., A. Bhatkar, and J. Nickerson. 1973. Predators of Solenopsis invicta queens prior to successful colony establishment. Environmental Entomology 2:1101-1103.

Wing, S. R. 1988. Cost of mating for female insects: risk of predation in Photinus collustrans (Coleoptera: Lampyridae). The American Naturalist 131:139-142.

\section{Figure legends}

Figure 1. Convergence stable virginity tendency strategies and the co-evolution of virginity tendency and mated queen sex ratio - (A) and (B): The solid lines illustrate the convergence stable virginity tendency strategy $u$ when mating carries a $20 \%$ mortality risk $(s=0.8)$, under queen controlled sex ratios in $(A)$, and under helper controlled sex ratios in (B). The convergence stable virginity tendency strategy is plotted as a function of the impact of mating and possible helpers on nest productivity (the 'production amplifier' $k$ ). The dashed line represents the breaking point for mated queen sex ratios. Under the dashed line, mated queens are producing both sons and daughters (as a function of both axis), and above it only daughters. The hollow line in (B) is a repeller for virginity tendency $u$. When the population average virginity tendency is below the repeller, virginity is selected against, and when above, virginity tendency is selected to converge to $1 / 2$. The arrows represent the direction of selection on virginity tendency when the population average is in those domains. (C), (D), (E) and (F): Streamplots for the co-evolution of virginity tendency $u$ and mated queen sex ratio $z$, under fixed production amplifiers and $20 \%$ mating mortality risk. The black arrows indicate the direction where selection will move a population from any combination of $u$ and $z$. Streamplots (C) and (D) are under queen controlled sex ratios and $(E)$ and $(F)$ under helper controlled sex ratios. The filled red dots represent the convergence points where stable population states are found.

This article is protected by copyright. All rights reserved. 
